\title{
Communications
}

\section{Multisite Recording of Extracellular Potentials Produced by Microchannel-Confined Neurons In-Vitro}

\author{
E. Claverol-Tinturé*, J. Cabestany, and X. Rosell
}

\begin{abstract}
Towards establishing electrical interfaces with patterned in vitro neurons, we have previously described the fabrication of hybrid elastomer-glass devices polymer-on-multielectrode array technology and obtained single-electrode recordings of extracellular potentials from confined neurons (Claverol-Tinturé et al., 2005). Here, we demonstrate the feasibility of spatially localized multisite recordings from individual microchannel-guided neurites extending from microwell-confined somas with good signal-to-noise ratios $(20 \mathrm{~dB})$ and spike magnitudes of up to $300 \mu \mathrm{V}$. Single-cell current source density (scCSD) analysis of the spatio-temporal patterns of membrane currents along individual processes is illustrated.
\end{abstract}

Index Terms-Cell patterning, current source density analysis, Helix aspersa, microfluidics, multichannel electrophysiology, neural interfaces, neuronal culture, PDMS, snail neurons.

\section{INTRODUCTION}

Multisite recording of neuronal activity with substrate integrated microelectrode arrays (MEAs) [2]-[4] is a promising alternative to the use of less scalable conventional electrophysiology [5]-[7] and photobleaching-prone voltage-sensitive dyes [8], [9]. In particular, deeper understanding of the complex relationship between cell morphology, active channel distribution and the associated spatio-temporal patterns of neuronal activity [10]-[13] could be obtained by the achievement of multisite MEA recordings from unambiguously identifiable compartments of neurons cultured in isolation.

Yet, the intrinsic anatomical plasticity of unconstrained developing cells results in continued changes in the relative position of the cellular compartments with respect to recording sites and, as a consequence, in unpredictable and time-changing signal-to-noise ratios. To address this issue and ensure long-term proximity between electrodes and neurons, efforts are focusing on the development of cell patterning techniques such as microcontact printing of adhesion promoting molecules [14], [15] and confinement by 3-D microstructures, e.g., fabricated by photo-thermal etching of wells and trenches in agarose layers [16], [17]. Recently, soft-lithography has emerged as a low-cost low-complexity technology [18] suitable for fabrication of polymeric microstructures of use in cell guiding. With this approach, patterning of mass neuronal cultures has been achieved [19]-[21] and, in conjunction with substrate integrated electrodes, single-site recordings from individual oocytes [22] and myocytes [23] have also been realized.

Manuscript received September 21, 2005; revised April 23, 2006. This work was supported in part by the Spanish Ministry for Science and Education through the Ramón y Cajal programme. Asterisk indicates corresponding author.

*E. Claverol-Tinturé is with the Department of Electronics, Technical University of Catalonia, Barcelona 08028, Spain. He is also with the Centre for Research in Biomedical Engineering (CREBEC), Barcelona 08028, Spain (e-mail: ectmail@eel.upc.edu).

J. Cabestany is with the Department of Electronics, Technical University of Catalonia, Barcelona 08028, Spain.

$\mathrm{X}$. Rosell is with the Department of Electronics, Technical University of Catalonia, Barcelona 08028, Spain. He is also with the Centre for Research in Biomedical Engineering (CREBEC), Barcelona 08028, Spain.

Digital Object Identifier 10.1109/TBME.2006.880903

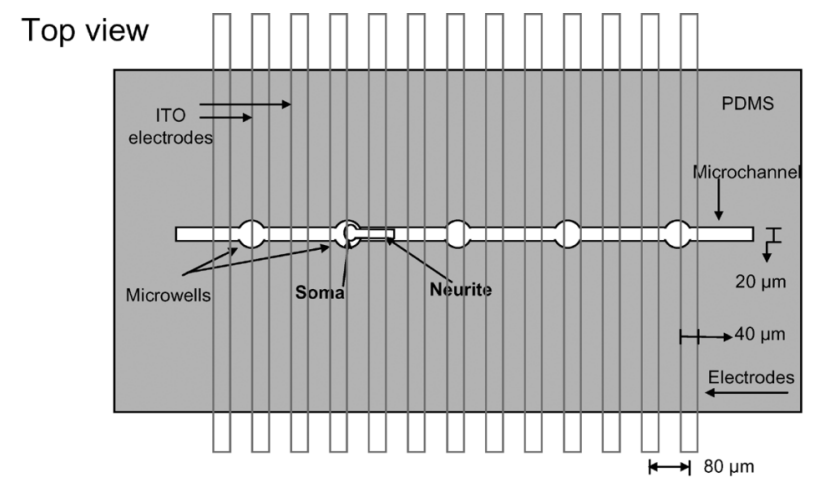

\section{Cross-section}

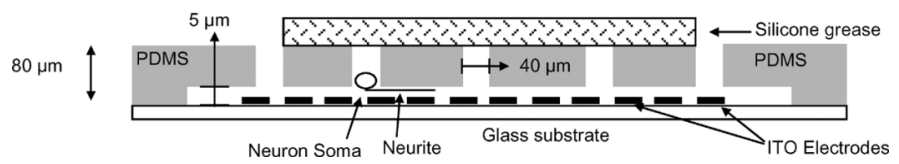

Fig. 1. Sketch of the multielectrode device, consisting of a sandwich structure assembled by overlaying a polymer film (60-80 $\mu \mathrm{m}$ thick) including microwells and microchannels on a planar multielectrode array [1]. The array was fabricated by photolithographically defining a set of parallel ITO electrodes on a glass substrate. Neurons were individually selected after tissue dissociation and positioned within microwells in contact with the poly-L-lysine treated glass substrate. As cells sproute neurites into the microchannels, the substrate embedded electrodes record extracellular potentials generated by active neurites.

We have previously reported on hybrid polymer-MEA devices, fabricated with a combination of soft-lithography and classic photolithography technologies, and demonstrated single-channel extracellular recordings from patterned neurites of isolated neurons [1]. Here we describe multi-point recordings from individual channel-confined neurites in vitro and illustrate the application of this technique to single-cell current source density analysis (scCSD).

\section{METHODS}

\section{A. Device Fabrication}

Fig. 1 shows a sketch of the device. A glass substrate with embedded parallel Indium-Tin-Oxide (ITO) electrodes was sandwiched with a PDMS film containing microwells connected by microchannels. Fabrication procedures have been described previously [1]. Briefly, ITO was sputtered on glass substrates to a resistivity of $80 \mathrm{ohm} / \mathrm{sq}$ or purchased with similar resistivity (PGO, Germany). The commercial substrates had a $\mathrm{SiO}_{2}$ barrier layer between glass and ITO. A set of 18 parallel electrodes, $40 \mu \mathrm{m}$ in width, were defined by standard photolithography techniques. The substrates were sonicated in acetone, isopropanol, neutral detergent and milli-Q grade water before assembly with elastomeric overlays. The overlays consisted of PDMS films (60-80 $\mu \mathrm{m}$ thick) with microcavities (40 $\mu \mathrm{m}$ in diameter) and microchannels (cross section $20 \times 5 \mu \mathrm{m}$ fabricated by microhole punching and soft-lithography from SU-8 masters [1] and laid on substrates with only coarse alignment. The sealed-well, as opposite to open-well, configuration was established by spreading silicone grease (Bayer, Germany) over the microwells to electrically insulate them.

The substrate was treated with poly-L-lysine $(0.1 \mathrm{mg} / \mathrm{ml})$ for $24 \mathrm{hr}$ prior to overlaying of the PDMS films in order to promote cell adhesion and growth. 
A

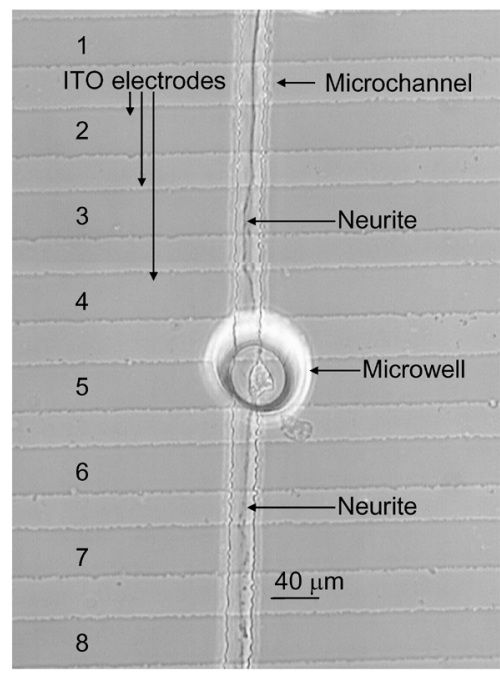

C
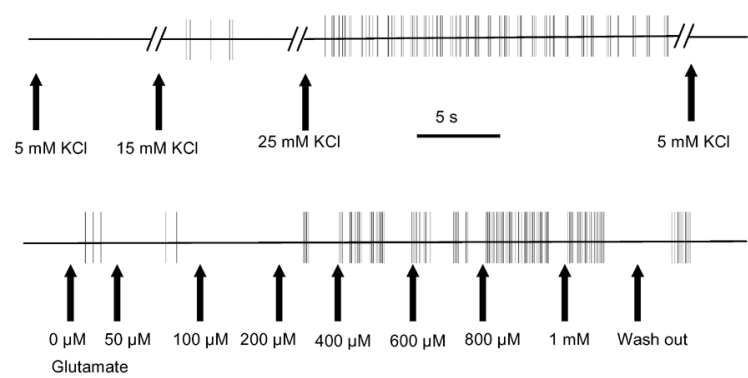

$12 \mathrm{~s}$
B
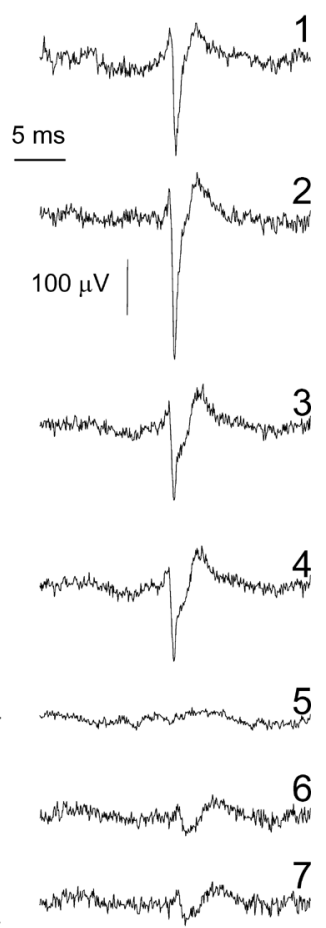

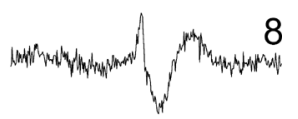

Fig. 2. (a) Phase-constrast image of a confined bipolar neuron after 12 days in vitro sprouting two neurites along a microchannel. (b) Extracellular potentials associated with an action potential and recorded by the eight microelectrodes shown in (a). (c) Raster-plot showing $\mathrm{KCl}$ and glutamate dose responses.

\section{B. Cell Culture}

The procedures to culture Helix aspersa neurons have been described previously [24]-[26]. Briefly, 4-6 week old snails (Cal Jep, Spain) were kept in hibernation until wetted 1 hour before the start of the dissection. Under anaesthesia by injection of $1 \mathrm{ml}$ of $0.08 \mathrm{mM} \mathrm{MgCl}_{2}$, the circumoesophageal ring was removed, trimmed free of surrounding tissue, cut in 8 pieces with fine scissors and transferred to culture medium (40\% L15 Leibovitz medium supplemented to the following concentrations: $62 \mathrm{mM} \mathrm{NaCl}, 5 \mathrm{mM} \mathrm{KCl}, 7 \mathrm{mM} \mathrm{CaCl}_{2}, 5 \mathrm{mM} \mathrm{MgCl}_{2}, 20 \mathrm{mM}$ HEPES, $10 \mathrm{mM}$ D-glucose, Peniciline 100 units/ml, Streptomycine $100 \mathrm{mg} / \mathrm{l}$, Fungizone $2.5 \mathrm{mg} / \mathrm{l}$, adjusted to $\mathrm{pH} 7.6$ with $\mathrm{NaOH}$ ). The ganglia were then torn with hypodermic needles to free them from the surrounding sheath and to extract individual neurons from the neuropiles.

After a 20 minute settling period, neurons with a diameter of 20 to 50 $\mu \mathrm{m}$ that had survived the procedure were identified under the dissection microscope, sucked with blunt-opening capillaries (0.58 mm ID, WPI, USA) and expelled into the wells.

As neurites extended beyond $100 \mu \mathrm{m}$ inside a microchannel (typically after several days in vitro), recordings were attempted. On occasions, spontaneous firing could be observed. When necessary, stimulation was usually realized by raising the extracellular concentration of $\mathrm{KCl}$ from $5 \mathrm{mM}$ to $25 \mathrm{mM}$. For Fig. 2, application of glutamate to the bath at concentrations ranging from $50 \mu \mathrm{M}$ up to $1 \mathrm{mM}$ was also used.

\section{Data Acquisition and Analysis}

A custom 16 channel amplifier (gain $\times 1300$, bandwidth $3.4 \mathrm{KHz}$ ) and a 12 bits ADC (6804E, National Instruments, 10 Ksamples/s per channel) were used for amplification and data acquisition. Of the 18 electrodes present in the device, numbers 1 and 18 were connected to the ground of the circuit. Electrode impedances at $1 \mathrm{KHz}$ were in the range 1.5-3 M $\Omega$, yielding recordings with noise levels below 10 $\mu \mathrm{V}_{\mathrm{rms}}$.

Offline filtering of line interference at $50 \mathrm{~Hz}$ and harmonics up to 350 $\mathrm{Hz}$ was implemented using Matlab (Mathworks, USA). However, good shielding often sufficed and software filters resulted in only minor improvements (10\% decrease in noise standard deviation). Accordingly, data were routinely used in raw format. To generate raster plots (Fig. 2), a threshold at $-100 \mu \mathrm{V}$ was used for spike detection.

To perform 1-D scCSD, the classical CSD formulation by Nicholson and Freeman [27] was adapted to microchannel-confined neurites. The membrane current per unit length above electrode $n, I_{m}(n)$, was calculated as

$$
I_{m}(n)=\frac{V_{e}(n+1)-2 V_{e}(n)+V_{e}(n-1)}{r_{e a} L^{2}}
$$

where $\mathrm{V}_{\mathrm{e}}(\mathrm{n})$ is the intrachannel potential at electrode $n, L$ is the center-to-center interelectrode distance $(80 \mu \mathrm{m})$, and $r_{e a}$ the intrachannel resistance per unit length. Single trial unaveraged raw data was used to generate scCSD profiles. To calculate $I_{m}(1)$ and $I_{m}(8)$ in Fig. 4, the intrachannel space located beyond the image was assumed grounded, i.e., $\mathrm{V}_{\mathrm{e}}(0)=0$ and $\mathrm{V}_{\mathrm{e}}(9)=0$, due to open wells present beyond the limits of the field of view.

\section{RESULTS}

Of the 35 neurons plated in microwells, 21 survived the procedure and attached to the poly-L-lysine coated substrate. Pharmacological 
A

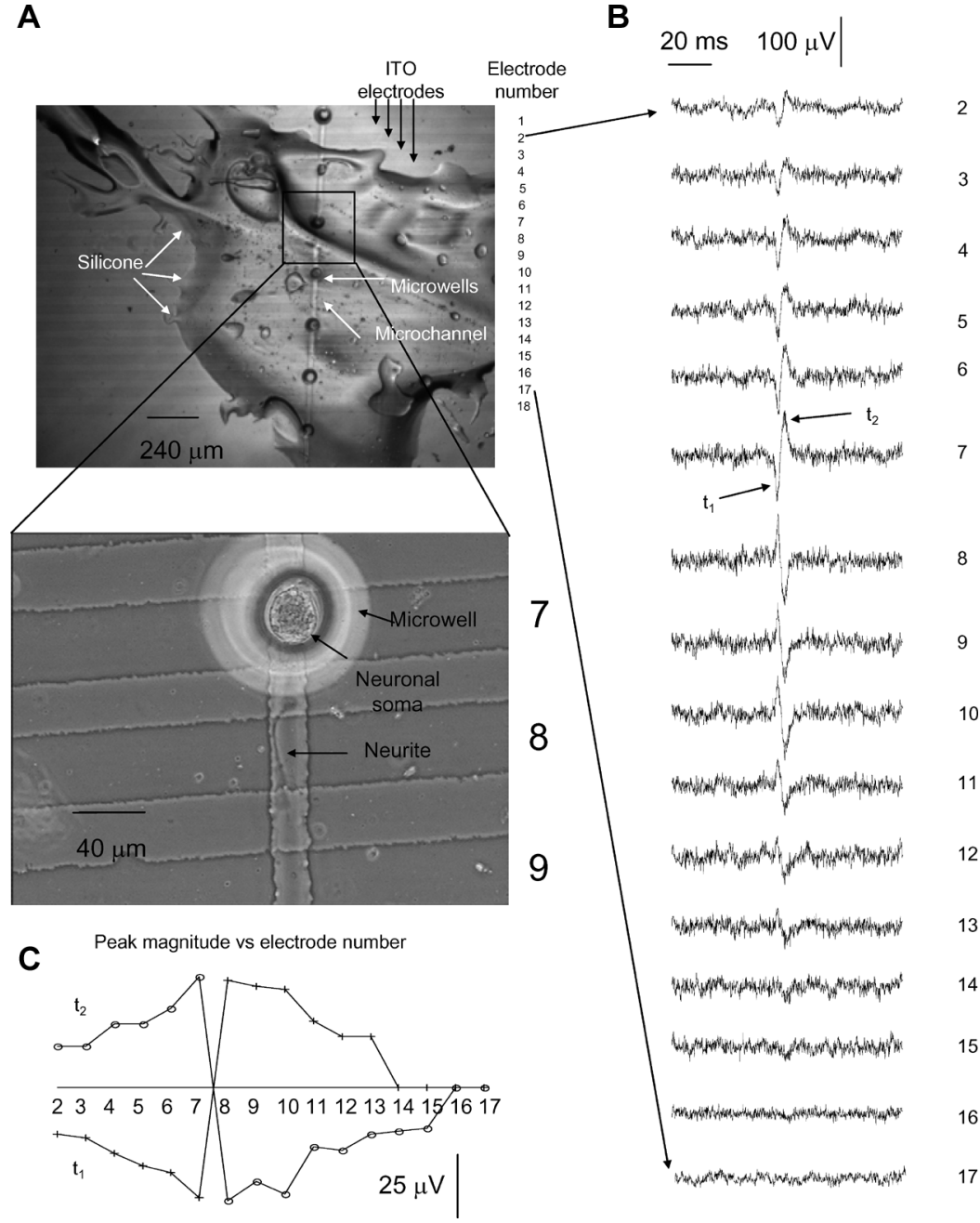

Fig. 3. (a) Phase-contrast image of a confined monopolar neuron with a short neurite outgrowth into the microchannel. The image (top) shows silicone covering the microwells and a close-up of the cell before sealing (bottom). (b) Extracellular potentials recorded by 18 electrodes, both local (7 and 8 ) and distal (2-6 and 9-17) with respect to ground (electrodes 1 and 18). (c) Spike magnitude versus electrode number at times $t_{1}$ and $t_{2}$.

stimulation was attempted on the 10 cells which grew microchannelconfined neurites of at least $100 \mu \mathrm{m}$ in length. Spikes were recorded from 9 neurons with magnitudes varying across cells and recordings sites and ranging from $30 \mu \mathrm{V}$ (barely detectable above noise levels) up to $300 \mu \mathrm{V}$.

Fig. 2(a) shows a microwell-confined neuron (after 12 days in culture) extending two neurites in opposite directions, each in excess of $200 \mu \mathrm{m}$ in length. Pharmacological stimulation with $\mathrm{KCl}(25 \mathrm{mM})$ and glutamate $(200 \mu \mathrm{M}-1 \mathrm{mM})$ were performed to elicit action potentials [Fig. 2(b)]. The largest spikes were recorded on electrode 2, with a mean peak of $-234.5 \pm 10.8 \mu \mathrm{V}$ and half-height width of $783.3 \pm 75.3 \mu \mathrm{s}$. Note that electrode 5 , although partially covered by the soma, remains electrically silent because the measurement was done in open-well configuration, effectively grounding the electrode. Fig. 2(c) shows the responses to increasing concentrations of $\mathrm{KCl}$ and glutamate as a test for the neuronal origin of the extracellular signals.

Fig. 3(a) shows a monopolar neuron within a device in sealed-well configuration (note silicone spread over the microwells). Its $100-\mu \mathrm{m}$ -long neurite generates $80 \mu \mathrm{V}_{\text {peak-to-peak }}$ biphasic spikes [Fig. 3(b)] recordable along the entire channel. Fig. 3(c) shows the spatial profile of the instantaneous potential at the two peaks, $t_{1}$ and $t_{2}$. Minima in the figure, i.e., zeros of the first spatial derivative, identify sinks and, conversely, maxima correspond to current sources. At time $t_{1}$, a fast inward cation flow, is located on electrode 7 , i.e., at or near the soma, concomitantly with a current source on electrode 8 , along the neurite and/or at the growth cone. At time $t_{2}$, the distribution of ion flows reverse, with a repolarizing outward source at the soma or hillock and a sink along the neurite and/or over the growth cone. Note that sealing the wells results in signals recorded at local as well as distal electrodes, decaying towards the (grounded) electrodes (numbers 1 and 18).

Intrachannel currents were calculated as $I_{n, n+1}=\left(V_{n}-\right.$ $\left.V_{n+1}\right) / R L$ where $\mathrm{R}$ is the channel resistance per unit length $(10.5$ $\mathrm{Kohm} / \mu \mathrm{m}), L$ is the separation between electrodes, and $\mathrm{V}_{\mathrm{n}}$ and $\mathrm{V}_{\mathrm{n}+1}$ are the potentials at two adjacent electrodes $n$ and $n+1$. Measured values were in the range $100-300 \mathrm{pA}(\mathrm{n}=9)$.

Neurons that extend neurites over at least 3 adjacent electrodes are suitable for scCSD. Fig. 4 shows CSD data corresponding to a spike generated by the cell shown in Fig. 2 (a single trial is shown without averaging). The maximum sink and source current densities were 3.7 $\mathrm{pA} / \mu \mathrm{m}$ and $2.78 \mathrm{pA} / \mu \mathrm{m}$. The earliest sink associated with the action potential appears in the proximity of electrode $7,150 \mu \mathrm{m}$ from the soma with a magnitude of $2 \mathrm{pA} / \mu \mathrm{m}$.

\section{DISCUSSION AND CONCLUSION}

We have described a technique for multisite recording of extracellular potentials from unequivocally identified neurite segments by 


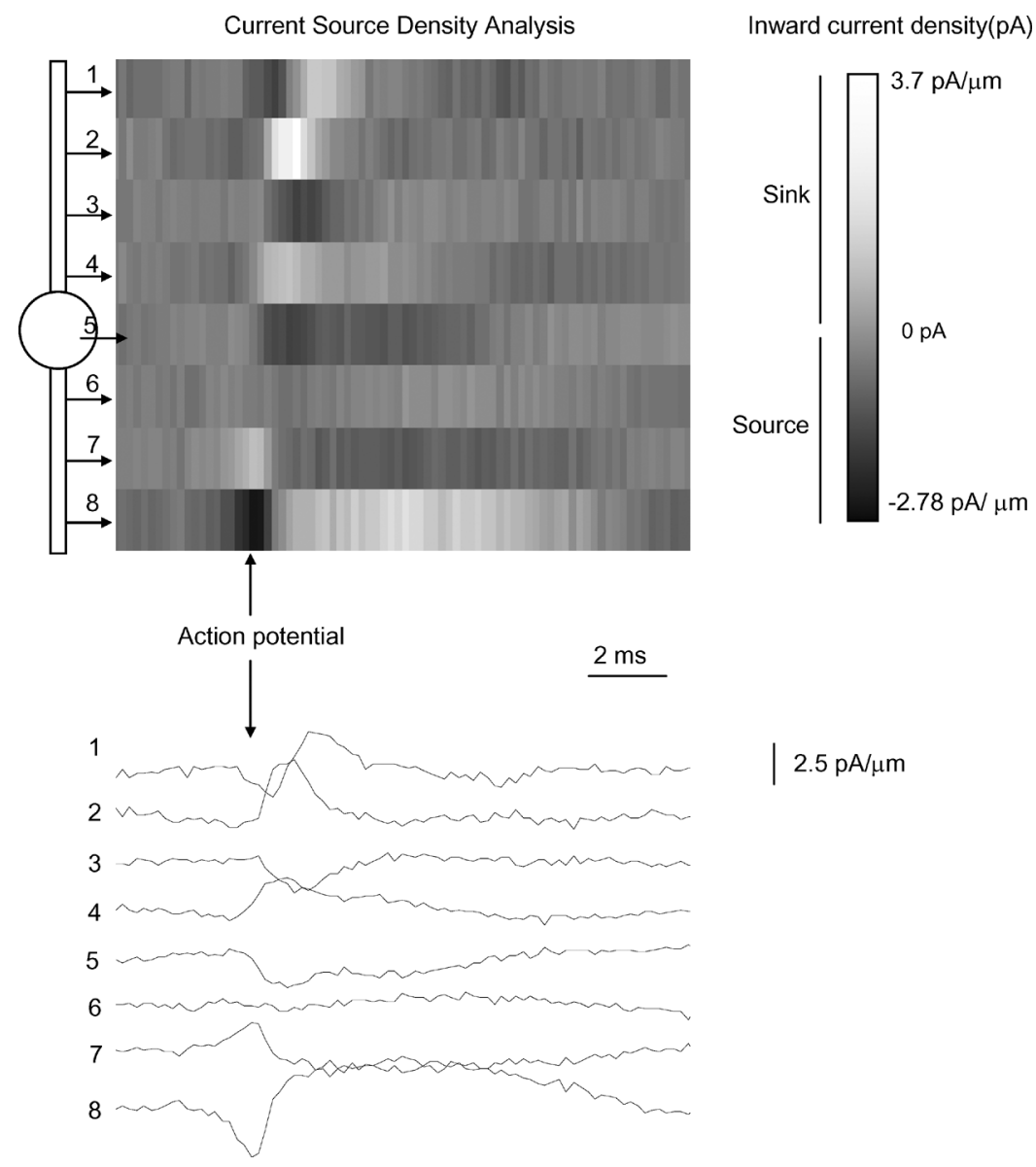

Fig. 4. scCSD corresponding to a spike produced by the neuron in Fig. 2.

means of hybrid polymer-multielectrode array devices. Embedded microwells and microchannels afford confinement of cells and ensure a known geometrical relationship between soma, neurites and electrodes. This is a promising strategy to increase the electrical stability of the electrode-neuron interface compared to conventional planar multielectrode arrays when used as substrates for culture of unrestrained neurons.

Physical confinement of and recording from somas has been previously achieved by others. For example, microfabricated polyimide pillars [28] can confine the cell body on a substrate embedded electrode to establish an electrical interface functionally similar to the loose cell-attached configuration [6]. Similarly, conic pores have been employed successfully to attain G $\Omega$ seals for somatic patch-clamp recordings [22]. Here we have demonstrated neurite patterning, multisite recording and scCSD in addition to soma confinement while making use of the low-complexity PoM approach to fabrication of 3-D microstructured devices.

The PoM technology, in its current state, can be of use in the context of neuronal biophysics research and drug screening. Further, minor refinements can enable it to support recordings from patterned networks in vitro.

\section{REFERENCES}

[1] E. Claverol-Tinturé, M. Ghirardi, F. Fiumara, X. Rosell, and J. Cabestany, "Multielectrode arrays with elastomeric microstructured overlays for extracellular recordings from patterned neurons," $J$. Neural Eng., vol. 2, no. 2, pp. 1-7, 2005.
[2] H. D. Mercer and R. L. White, "Photolithographic fabrication and physiological performance of microelectrode arrays for neural stimulation," IEEE Trans. Biomed. Eng., vol. BME-25, no. 6, pp. 494-500, Jun. 1978.

[3] G. W. Gross, "Simultaneous single unit recording in vitro with a photoetched laser deinsulated gold multimicroelectrode surface," IEEE Trans. Biomed. Eng., vol. BME-26, no. 5, pp. 273-279, May 1979.

[4] C. D. James, A. J. Spence, N. M. Dowell-Mesfin, R. J. Hussain, K. L. Smith, H. G. Craighead, M. S. Isaacson, W. Shain, and J. N. Turner, "Extracellular recordings from patterned neuronal networks using planar microelectrode arrays," IEEE Trans. Biomed Eng., vol. 51, no. 9, pp. 1640-1648, Sep. 2004.

[5] R. M. Fitzsimonds, H. Song, and M. Poo, "Propagation of activitydependent synaptic depression in simple neural networks," Nature, vol. 388, pp. 439-448, 1997.

[6] B. Barbour and P. Isope, "Combining loose cell-attached stimulation and recording," J. Neurosci. Meth., vol. 103, pp. 199-208, 2000.

[7] E. Claverol-Tinturé and J. Pine, "Extracellular potentials in low-density dissociated neuronal cultures," J Neurosci. Meth., vol. 117, no. 1, pp. $13-21,2002$.

[8] L. Cohen, "Optical monitoring of activity in the nervous system: a brief history," Tsitologiia, vol. 37, no. 12, pp. 1136-1141, 1995.

[9] W. Jin, R. J. Zhang, and J. Y. Wu, "Voltage-sensitive dye imaging of population neuronal activity in cortical tissue," J. Neurosci. Meth., vol. 115 , no. 1, pp. 13-27, 2002.

[10] C. Koch and I. Segev, Methods in Neural Modeling: From Ions to Networks. Cambridge, MA: The MIT Press, 1998.

[11] D. Pinault, "Backpropagation of action potentials generated at ectopic axonal loci: hypothesis that axon terminals integrate local environmental signals," Brain Res. Brain Res. Rev., vol. 21, no. 1, pp. 42-92, 1995.

[12] S. Cash and R. Yuste, "Input summation by cultured pyramidal neurons is linear and position-independent," J. Neurosci., vol. 18, no. 1, pp. $10-15,1998$. 
[13] A. T. Gulledge, B. M. Kampa, and G. J. Stuart, "Synaptic integration in dendritic trees," J. Neurobiol., vol. 64, no. 1, pp. 75-90, 2005.

[14] J. C. Chang, G. J. Brewer, and B. C. Wheeler, "A modified microstamping technique enhances polylysine transfer and neuronal cell patterning," Biomaterials, vol. 24, no. 17, pp. 2863-2870, 2003.

[15] C. D. James, A. J. Spence, N. M. Dowell-Mesfin, R. J. Hussain, K. L. Smith, H. G. Craighead, M. S. Isaacson, W. Shain, and J. N. Turner, "Extracellular recordings from patterned neuronal networks using planar microelectrode arrays," IEEE Trans. Biomed. Eng., vol. 51, no. 9, pp. 1640-1648, Sep. 2004.

[16] H. Moriguchi, K. Takahashi, Y. Sugio, Y. Wakamoto, I. Inoue, Y. Jimbo, and K. Yasuda, "On-chip neural cell cultivation using agarosemicrochamber array constructed by a photothermal etching method," Electr. Eng. Japan, vol. 146, no. 2, pp. 153-1458, 2004.

[17] I. Suzuki, Y. Sugio, H. Moriguchi, Y. Jimbo, and K. Yasuda, "Modification of a neuronal network direction using stepwise photo-thermal etching of an agarose architecture," J Nanobiotech., vol. 2, no. 1, p. 7, 2004.

[18] Y. Xia and G. M. Whitesides, "Soft lithography," Ann. Rev. Mater. Sci., vol. 28, pp. 153-184, 1998.

[19] L. Griscom, P. Degenaar, B. LePioufle, E. Tamiya, and H. Fujita, "Cell placement and neural guidance using a three-dimensional microfluidic array," Jpn. J. Appl. Phys., vol. 40, pp. 5485-5490, 2001.

[20] A. M. Taylor, S. W. Rhee, C. H. Tu, D. H. Cribbs, C. W. Cotman, and N. L. Jeon, "Microfluidic multicompartment device for neuroscience research," Langmuir, vol. 19, pp. 1551-1556, 2003.

[21] S. W. Rhee, A. M. Taylor, C. H. Tu, D. H. Cribbs, C. W. Cotman, and N. L. Jeon, "Patterned cell culture inside microfluidic devices," Lab Chip, vol. 4, 2004.

[22] K. G. Klemic, J. F. Klemic, M. A. Reed, and F. J. Sigworth, "Micromolded PDMS planar electrode allows patch clamp electrical recordings from cells," Biosensors Bioelectron., vol. 17, pp. 597-604, 2002.

[23] N. Klauke, G. L. Smith, and J. Cooper, "Stimulation of single isolated adult ventricular myocytes within a low volume using planar microelectrode array," Biophys. J., vol. 85, no. 3, pp. 1766-1774, 2003.

[24] K. A. Green, B. Powell, and G. A. Cottrell, "Unitary K+ currents in growth cones and perikaryon of identified Helix neurones in culture," J. Exper. Biol., vol. 149, pp. 79-94, 1990.

[25] S. K. Tiwari and M. L. Woodruff, "Helix aspersa neurones maitain vigorous electrical activity when co-cultured with ntact H. Aspersa," Comp. Boche. Physol. [C], vol. 101, pp. 163-174, 1992.

[26] M. Ghirardi, A. Casadio, L. Santarelli, and P. G. Montarolo, "Aplysia hemolymph promotes neurite outgrowth and synaptogenesis of identified Helix neurons in cell culture," Invert Neurosci., vol. 2, no. 1, pp. 41-49, 1996.

[27] C. Nicholson and J. A. Freeman, "Theory of current source-density analysis and determination of conductivity tensor for anuran cerebellum," J. Neurophysiol., vol. 38, no. 2, pp. 356-368, 1975.

[28] G. Zeck and P. Fromherz, "Noninvasive neuroelectronic interfacing with synaptically connected snail neurons immobilized on a semiconductor chip," Proc. Nat. Acad. Sci. USA, vol. 98, no. 18, pp. 10457-10462, 2001

\section{Design of Surface Electrode Array for Measuring Conduction Velocity in the Human Genioglossus Muscle}

Ciara M. O'Connor*, Lize der van Merwe, Simon Langran, Michael O'Sullivan, Philip Nolan, Christopher L. Vaughan, and Mark J. O'Malley

Abstract-A new appliance, incorporating linear arrays of pin electrodes for genioglossus (GG) surface electromyography measurement, is presented. This design enables the estimation of GG muscle fiber conduction velocity, which decreases with fatigue. The performance of the device was evaluated for ten healthy human subjects during fatiguing and force varying contractions.

Index Terms-Conduction velocity estimation, electromyography, genioglossus muscles, linear electrode array, obstructive sleep apnea syndrome.

\section{INTRODUCTION}

THE genioglossus (GG) muscles are the primary protrudors of the tongue and play a vital role in maintaining the patency of the upper airway, particularly during sleep. In patients with obstructive sleep apnea syndrome (OSAS), a common and serious medical disorder [1], the upper airway collapses repeatedly during sleep causing a cessation of airflow terminated only by an arousal from sleep. Abnormalities in GG activation, function and structure have been reported for OSAS patients [2] and the complex pathogenesis of OSAS, in particular the role of fatigue, is still incompletely understood [3].

Information on the state of a muscle can be gleaned using surface electromyography (SEMG) to track the muscle fiber conduction velocity (CV). The use of a linear electrode array facilitates CV estimation as well as the determination of anatomical properties such as the locations of innervation zones of muscles and optimal locations for SEMG electrode placement [4]. CV tends to decrease with fatigue, and indirect myoelectrical manifestations of fatigue include a shift towards lower frequency in power spectrum of the signal and an increase in the amplitude of the signal, which are attributed in part to the change in $\mathrm{CV}$, as well as other factors [5]. The most widely used electrode configuration for GG SEMG recording was introduced by Doble et al. [6]. This electrode configuration records the differential signal between the two GG muscles, using two bar electrodes, located one on either side of the midline of the mouth. While this configuration would be suitable for recording muscle activation times, it could not be used to measure $\mathrm{CV}$. Our research has previously indicated that an adaptation of this

Manuscript received October 27, 2005; revised May 21, 2006. This work was supported in part by the UCD Conway Institute of Biomolecular and Biomedical Research, in part by Enterprise Ireland, in part by The Wellcome Trust, and in part the School of Electrical, Electronic and Mechanical Engineering, University College Dublin. Asterisk indicates corresponding author.

*C. M. O'Connor is with the School of Electrical, Electronic and Mechanical Engineering, University College Dublin, Belfield, Dublin 4, Ireland (e-mail: ciara@ee.ucd.ie).

L. van der Merwe is with the Biostatistics Unit, Medical Research Council of South Africa, 7505 Tygerberg, South Africa.

S. Langran and M. O'Sullivan are with the Department of Restorative Dentistry and Periodontology, Dublin Dental School and Hospital, Trinity College, Dublin 4, Ireland.

P. Nolan was with the School of Medicine and Medical Science, University College Dublin, Ireland. He is now with the Department of Academic Affairs, University College Dublin, Dublin 4, Ireland.

C. L. Vaughan is with the MRC/UCT Medical Imaging Research Unit, Department of Human Biology, Faculty of Health Sciences, University of Cape Town, Western Cape 7925, South Africa.

M. J. O'Malley is with the School of Electrical, Electronic and Mechanical Engineering, University College Dublin, Dublin 4, Ireland.

Digital Object Identifier 10.1109/TBME.2006.880911 